Honam Mathematical J. 35 (2013), No. 4, pp. 551-564

http://dx.doi.org/10.5831/HMJ.2013.35.4.551

\title{
FIXED POINT THEOREMS IN MENGER SPACES USING AN IMPLICIT RELATION
}

\author{
Sunny Chauhan*, M. Alamgir Khan and B. D. Pant
}

\begin{abstract}
In 2008, Al-Thagafi and Shahzad [Generalized I-nonexpansive selfmaps and invariant approximations, Acta Math. Sinica, $24(5)$ (2008), 867-876] introduced the notion of occasionally weakly compatible mappings in metric spaces. In this paper, we prove some common fixed point theorems for families of occasionally weakly compatible mappings in Menger spaces using an implicit relation. We also give an illustrative example to support our main result.
\end{abstract}

\section{Introduction}

The concept of probabilistic metric space was first introduced and studied by Menger [30], which is a generalization of the metric space. The study of this space was expanded rapidly with the pioneering works of Schweizer and Sklar [40,41] and some of their coworkers. Such a probabilistic generalization of metric spaces appears to be well adapted for the investigation of physiological thresholds and physical quantities. It is also of fundamental importance in probabilistic functional analysis, nonlinear analysis and applications (see [18, 19, 24]). In 1972, Sehgal and Bharucha-Reid [42] initiated the study of contraction mappings in probabilistic metric spaces (briefly, PM-spaces) which is an important concept in the development of fixed point theorems.

Jungck and Rhoades [26] introduced the notion of weakly compatible mappings in metric spaces. Singh and Jain [45] formulated the notion of weakly compatible mappings in probabilistic settings and proved some fixed point theorems in Menger spaces. In 2008, Al-Thagafi and Shahzad [7] introduced the notion of occasionally weakly compatible (briefly, owc)

Received September 7, 2012. Accepted April 25, 2013.

2010 Mathematics Subject Classification. Primary 47H10; Secondary 54H25.

Key words and phrases. $t$-norm, Menger space, occasionally weakly compatible mappings, implicit relation, fixed point.

${ }^{*}$ Corresponding author 
mappings in metric spaces, while Chandra and Bhatt [17] extended the notion of owc mappings in probabilistic setting. It is worth to mention that every pair of weak compatible self mappings is owc but the converse is not always true. Many authors proved common fixed point theorems using the notion of owc mappings in different settings (see $[1,2,3,4,5$, $6,7,8,10,11,12,13,14,15,16,17,20,21,22,23,27,28,29,32,33$, $34,35,36,39,46])$.

In 1999, Popa used the family of implicit real functions and proved some common fixed point theorems (see [37, 38]). These observations motivated us to prove common fixed point theorems for families of owc mappings in Menger spaces. We also give an example to support our main result.

\section{Preliminaries}

Definition 2.1. [41] A mapping $\triangle:[0,1] \times[0,1] \rightarrow[0,1]$ is $t$-norm if $\triangle$ is satisfying the following conditions:

(1) $\triangle$ is commutative and associative,

(2) $\triangle(a, 1)=a$ for all $a \in[0,1]$

(3) $\triangle(a, b) \leq \triangle(c, d)$ whenever $a \leq c$ and $b \leq d$ and $a, b, c, d \in[0,1]$.

The following are the basic $t$-norms:

$$
\begin{aligned}
\triangle(a, b) & =\min \{a, b\}, \\
\triangle(a, b) & =a b, \\
\triangle(a, b) & =\max \{a+b-1,0\} .
\end{aligned}
$$

Definition 2.2. [41] A mapping $F: \mathbb{R} \rightarrow \mathbb{R}^{+}$is called a distribution function if it is non-decreasing and left continuous with $\inf \{F(t): t \in$ $\mathbb{R}\}=0$ and $\sup \{F(t): t \in \mathbb{R}\}=1$.

We shall denote by $\Im$ the set of all distribution functions defined on $(-\infty, \infty)$ while $H(t)$ will always denote the specific distribution function defined by

$$
H(t)= \begin{cases}0, & \text { if } t \leq 0 \\ 1, & \text { if } t>0\end{cases}
$$

If $X$ is a non-empty set, $\mathcal{F}: X \times X \rightarrow \Im$ is called a probabilistic distance on $X$ and the value of $\mathcal{F}$ at $(x, y) \in X \times X$ is represented by $F_{x, y}$.

Definition 2.3. [41] A PM-space is an ordered pair $(X, \mathcal{F})$, where $X$ is a nonempty set of elements and $\mathcal{F}$ is a probabilistic distance satisfying the following conditions: for all $x, y, z \in X$ and $t, s>0$, 
(1) $F_{x, y}(t)=H(t)$ for all $t>0$ if and only $x=y$,

(2) $F_{x, y}(0)=0$

(3) $F_{x, y}(t)=F_{y, x}(t)$,

(4) if $F_{x, y}(t)=1$ and $F_{y, z}(s)=1$ then $F_{x, z}(t+s)=1$.

The ordered triplet $(X, \mathcal{F}, \triangle)$ is called a Menger space if $(X, \mathcal{F})$ is a PM-space, $\triangle$ is a $t$-norm and the following inequality holds:

$$
F_{x, y}(t+s) \geq \triangle\left(F_{x, z}(t), F_{z, y}(s)\right),
$$

for all $x, y, z \in X$ and $t, s>0$.

Every metric space $(X, d)$ can always be realized as a PM-space by considering $\mathcal{F}: X \times X \rightarrow \Im$ defined by $F_{x, y}(t)=H(t-d(x, y))$ for all $x, y \in X$. So PM-spaces offer a wider framework than that of metric spaces and are better suited to cover even wider statistical situations.

Definition 2.4. [45] A pair $(A, S)$ of self mappings of a Menger space $(X, \mathcal{F}, \triangle)$ is said to be weakly compatible (or coincidentally commuting) if they commute at their coincidence points, that is, if $A x=S x$ for some $x \in X$, then $A S x=S A x$.

The concept of owc mappings due to [7] is a proper generalization of nontrivial weakly compatible mappings which do have a coincidence point. The counterpart of the concept of owc maps in PM-spaces is as follows:

Definition 2.5. A pair $(A, S)$ of self mappings of a Menger space $(X, \mathcal{F}, \triangle)$ is owc if and only if there is a point $x \in X$ which is a coincidence point of $A$ and $S$ at which $A$ and $S$ commute.

From the following example it is clear that owc is more general than weak compatibility.

Example 2.1. Let $(X, \mathcal{F}, \triangle)$ be a Menger space, where $X=\mathbb{R}$ and

$$
F_{x, y}(t)= \begin{cases}\frac{t}{t+|x-y|}, & \text { if } t>0 ; \\ 0, & \text { if } t=0 .\end{cases}
$$

Define $A, S: \mathbb{R} \rightarrow \mathbb{R}$ by $A x=3 x$ and $S x=x^{2}$ for all $x \in \mathbb{R}$. Then $A x=S x$ for $x=0,3$ but $A S(0)=S A(0)$, and $A S(3) \neq S A(3)$. Thus $A$ and $S$ are owc mappings but not weakly compatible.

Lemma 2.1. [27] Let $(X, \mathcal{F}, \triangle)$ be a Menger space, $A$ and $S$ are owc self mappings of $X$. If $A$ and $S$ have a unique point of coincidence, $w=A x=S x$, then $w$ is the unique common fixed point of $A$ and $S$. 


\section{Implicit Relation}

Many authors proved a number of common fixed point theorems using the notion of implicit relation on different spaces (see [9], [25], [37], [38], [44]). Recently, Sedghi et al. [43] proved a common fixed point theorem in fuzzy metric spaces by using the following implicit relation:

Let $\mathcal{T}$ be the set of all continuous functions $T:[0,1]^{5} \rightarrow[-1,1]$ satisfying the following conditions:

$\left(T_{1}\right) T\left(t_{1}, \ldots, t_{5}\right)$ is increasing in $t_{1}$ and decreasing in $t_{2}, \ldots, t_{5}$.

$\left(T_{2}\right) T(u, v, v, v, v) \geq 0$ implies that $u>v, \forall v \in[0,1)$ and $\forall u \in[0,1]$.

Remark 3.1. [43] It is easy to see that $T(v, v, v, v, v) \geq 0$ implies that $v=1$. If $v \neq 1$, by $\left(T_{2}\right), T(v, v, v, v, v) \geq 0$ implies that $v>v$, is a contradiction. Thus $v=1$.

Example 3.1. [43] Let $T:[0,1]^{5} \rightarrow[-1,1]$ be defined by $T\left(t_{1}, t_{2}, t_{3}\right.$, $\left.t_{4}, t_{5}\right)=t_{1}-\left(\min \left\{t_{2}, t_{3}, t_{4}, t_{5}\right\}\right)^{h}$ for some $0<h<1$.

\section{Results}

In this section, first we prove a common fixed point theorem for any even number of owc mappings in Menger space employing an implicit relation.

Theorem 4.1. Let $P_{1}, P_{2}, \ldots, P_{2 n}, A$ and $B$ be self mappings of a Menger space $(X, \mathcal{F}, \triangle)$ with $\triangle(a, a)=a$ for all $a \in[0,1]$ satisfying the following conditions:

(1) there exists $k \in(0,1)$ and $T \in \mathcal{T}$ such that

$$
\begin{aligned}
& T\left(\begin{array}{c}
F_{A x, B y}(k t), F_{P_{1} P_{3} \ldots P_{2 n-1} x, P_{2} P_{4} \ldots P_{2 n} y}(t), \\
F_{A x, P_{1} P_{3} \ldots P_{2 n-1} x}(t), F_{B y, P_{2} P_{4} \ldots P_{2 n} y}(t), \\
\triangle\left(F_{B y, P_{1} P_{3} \ldots P_{2 n-1} x}(\alpha t), F_{A x, P_{2} P_{4} \ldots P_{2 n} y}(2 t-\alpha t)\right)
\end{array}\right) \geq 0, \\
& \quad \text { for all } x, y \in X, \alpha \in(0,2) \text { and } t>0 .
\end{aligned}
$$


(2) Suppose that

$$
\left\{\begin{aligned}
P_{1}\left(P_{3} \ldots P_{2 n-1}\right) & =\left(P_{3} \ldots P_{2 n-1}\right) P_{1}, \\
P_{1} P_{3}\left(P_{5} \ldots P_{2 n-1}\right) & =\left(P_{5} \ldots P_{2 n-1}\right) P_{1} P_{3}, \\
& \vdots \\
P_{1} \ldots P_{2 n-3}\left(P_{2 n-1}\right) & =\left(P_{2 n-1}\right) P_{1} \ldots P_{2 n-3}, \\
A\left(P_{3} \ldots P_{2 n-1}\right) & =\left(P_{3} \ldots P_{2 n-1}\right) A, \\
A\left(P_{5} \ldots P_{2 n-1}\right) & =\left(P_{5} \ldots P_{2 n-1}\right) A, \\
& \vdots \\
A P_{2 n-1} & =P_{2 n-1} A, \\
P_{2}\left(P_{4} \ldots P_{2 n}\right) & =\left(P_{4} \ldots P_{2 n}\right) P_{2}, \\
P_{2} P_{4}\left(P_{6} \ldots P_{2 n}\right) & =\left(P_{6} \ldots P_{2 n}\right) P_{2} P_{4}, \\
& \vdots \\
P_{2} \ldots P_{2 n-2}\left(P_{2 n}\right) & =\left(P_{2 n}\right) P_{2} \ldots P_{2 n-2}, \\
B\left(P_{4} \ldots P_{2 n}\right) & =\left(P_{4} \ldots P_{2 n}\right) B, \\
B\left(P_{6} \ldots P_{2 n}\right) & =\left(P_{6} \ldots P_{2 n}\right) B, \\
& \vdots \\
B P_{2 n} & =P_{2 n} B
\end{aligned}\right\}
$$

Then, if the pairs $\left(A, P_{1} P_{3} \ldots P_{2 n-1}\right)$ and $\left(B, P_{2} P_{4} \ldots P_{2 n}\right)$ are each owc, it follows that $P_{1}, P_{2}, \ldots, P_{2 n}, A$ and $B$ have a unique common fixed point in $X$.

Proof. Since the pairs $\left(A, P_{1} P_{3} \ldots P_{2 n-1}\right)$ and $\left(B, P_{2} P_{4} \ldots P_{2 n}\right)$ are each owc, there exist points $u, v \in X$ such that $A u=P_{1} P_{3} \ldots P_{2 n-1} u$, $A\left(P_{1} P_{3} \ldots P_{2 n-1}\right) u=\left(P_{1} P_{3} \ldots P_{2 n-1}\right) A u$ and $B v=P_{2} P_{4} \ldots P_{2 n} v, B\left(P_{2}\right.$ $\left.P_{4} \ldots P_{2 n}\right) v=\left(P_{2} P_{4} \ldots P_{2 n}\right) B v$. Now we assert that $A u=B v$. Putting $x=u, y=v$ and $\alpha=1$ in inequality (1), we get

$$
\begin{gathered}
T\left(\begin{array}{c}
F_{A u, B v}(k t), F_{P_{1} P_{3} \ldots P_{2 n-1} u, P_{2} P_{4} \ldots P_{2 n} v}(t), \\
F_{A u, P_{1} P_{3} \ldots P_{2 n-1} u}(t), F_{B v, P_{2} P_{4} \ldots P_{2 n} v}(t), \\
\triangle\left(F_{B v, P_{1} P_{3} \ldots P_{2 n-1} u}(t), F_{A u, P_{2} P_{4} \ldots P_{2 n} v}(t)\right)
\end{array}\right) \geq 0, \\
T\left(\begin{array}{c}
F_{A u, B v}(k t), F_{A u, B v}(t), F_{A u, A u}(t), \\
F_{B v, B v}(t), \triangle\left(F_{B v, A u}(t), F_{A u, B v}(t)\right)
\end{array}\right) \geq 0 .
\end{gathered}
$$

Since $T$ is increasing in $t_{1}$ and decreasing in $t_{2}, \ldots, t_{5}$, we get

$$
T\left(F_{A u, B v}(t), F_{A u, B v}(t), F_{A u, B v}(t), F_{A u, B v}(t), F_{A u, B v}(t)\right) \geq 0 .
$$

Thus by Remark 3.1, we have $F_{A u, B v}(t)=1$. Hence $A u=B v$. Moreover, if there is another point $z$ such that $A z=\left(P_{1} P_{3} \ldots P_{2 n-1}\right) z$. Then using inequality (1), it follows that $A z=\left(P_{1} P_{3} \ldots P_{2 n-1}\right) z=B v=$ $\left(P_{2} P_{4} \ldots P_{2 n}\right) v$, or $A u=A z$. Hence $w=A u=\left(P_{1} P_{3} \ldots P_{2 n-1}\right) u$ is the 
unique point of coincidence of $A$ and $P_{1} P_{3} \ldots P_{2 n-1}$. By Lemma 2.1, it follows that $w$ is the unique common fixed point of $A$ and $P_{1} P_{3} \ldots P_{2 n-1}$. By symmetry, $q=B v=\left(P_{2} P_{4} \ldots P_{2 n}\right) v$ is the unique common fixed point of $B$ and $P_{2} P_{4} \ldots P_{2 n}$. Since $w=q$, we obtain that $w$ is the unique common fixed point of $B$ and $P_{2} P_{4} \ldots P_{2 n}$. Now we show that $w$ is the fixed point of all the component mappings. By putting $x=$ $\left(P_{3} \ldots P_{2 n-1}\right) w, y=w, \alpha=1, P_{1}^{\prime}=P_{1} P_{3} \ldots P_{2 n-1}$ and $P_{2}^{\prime}=P_{2} P_{4} \ldots P_{2 n}$ in inequality (1), we have

$$
\begin{gathered}
T\left(\begin{array}{c}
F_{A P_{3} \ldots P_{2 n-1} w, B w}(k t), F_{P_{1}^{\prime} P_{3} \ldots P_{2 n-1} w, P_{2}^{\prime} w}(t), \\
F_{A P_{3} \ldots P_{2 n-1} w, P_{1}^{\prime} P_{3} \ldots P_{2 n-1} w}(t), F_{B w, P_{2}^{\prime} w}(t), \\
\triangle\left(F_{B w, P_{1}^{\prime} P_{3} \ldots P_{2 n-1} w}(t), F_{A P_{3} \ldots P_{2 n-1} w, P_{2}^{\prime} w(t)}\right)
\end{array}\right) \geq 0 \\
T\left(\begin{array}{c}
F_{P_{3} \ldots P_{2 n-1} w, w}(k t), F_{P_{3} \ldots P_{2 n-1} w, w}(t), \\
F_{P_{3} \ldots P_{2 n-1} w, P_{3} \ldots P_{2 n-1} w}(t), F_{w, w}(t), \\
\triangle\left(F_{w, P_{3} \ldots P_{2 n-1} w}(t), F_{P_{3} \ldots P_{2 n-1} w, w}(t)\right)
\end{array}\right) \geq 0 .
\end{gathered}
$$

Since $T$ is increasing in $t_{1}$ and decreasing in $t_{2}, \ldots, t_{5}$, we get

$$
T\left(\begin{array}{c}
F_{P_{3} \ldots P_{2 n-1} w, w}(t), F_{P_{3} \ldots P_{2 n-1} w, w}(t), F_{P_{3} \ldots P_{2 n-1} w, w}(t), \\
F_{P_{3} \ldots P_{2 n-1} w, w}(t), F_{P_{3} \ldots P_{2 n-1} w, w}(t)
\end{array}\right) \geq 0 .
$$

Thus by Remark 3.1, we have $F_{P_{3} \ldots P_{2 n-1} w, w}(t)=1$ that is $P_{3} \ldots P_{2 n-1}$ $w=w$. Hence, $P_{1} w=w$. Continuing this procedure, we have

$A w=P_{1} w=P_{3} w=\ldots=P_{2 n-1} w=w$.

So,

$B w=P_{2} w=P_{4} w=\ldots=P_{2 n} w=w$.

That is, $w$ is the unique common fixed point of $P_{1}, P_{2}, \ldots, P_{2 n}, A$ and $B$.

The following result is a slight generalization of Theorem 4.1.

Corollary 4.1. Let $\left\{L_{\zeta}\right\}_{\zeta \in J}$ and $\left\{P_{i}\right\}_{i=1}^{2 n}$ be two families of self mappings of a Menger space $(X, \mathcal{F}, \triangle)$ with $\triangle(a, a)=a$ for all $a \in[0,1]$ satisfying the following conditions:

(1) there exists a fixed $\eta \in J, k \in(0,1)$ and $T \in \mathcal{T}$ such that

$$
T\left(\begin{array}{c}
F_{L_{\zeta} x, L_{\eta} y}(k t), F_{P_{1} P_{3} \ldots P_{2 n-1} x, P_{2} P_{4} \ldots P_{2 n} y}(t), \\
F_{L_{\zeta} x, P_{1} P_{3} \ldots P_{2 n-1} x}(t), F_{L_{\eta} y, P_{2} P_{4} \ldots P_{2 n} y}(t), \\
\triangle\left(F_{L_{\eta} y, P_{1} P_{3} \ldots P_{2 n-1} x}(\alpha t), F_{L_{\zeta} x, P_{2} P_{4} \ldots P_{2 n} y}(2 t-\alpha t)\right)
\end{array}\right) \geq 0,
$$

for all $x, y \in X, \alpha \in(0,2)$ and $t>0$. 
(2) Suppose that

$$
\left\{\begin{aligned}
P_{1}\left(P_{3} \ldots P_{2 n-1}\right) & =\left(P_{3} \ldots P_{2 n-1}\right) P_{1}, \\
P_{1} P_{3}\left(P_{5} \ldots P_{2 n-1}\right) & =\left(P_{5} \ldots P_{2 n-1}\right) P_{1} P_{3}, \\
& \vdots \\
P_{1} \ldots P_{2 n-3}\left(P_{2 n-1}\right) & =\left(P_{2 n-1}\right) P_{1} \ldots P_{2 n-3}, \\
L_{\zeta}\left(P_{3} \ldots P_{2 n-1}\right) & =\left(P_{3} \ldots P_{2 n-1}\right) L_{\zeta}, \\
L_{\zeta}\left(P_{5} \ldots P_{2 n-1}\right) & =\left(P_{5} \ldots P_{2 n-1}\right) L_{\zeta}, \\
& \vdots \\
L_{\zeta} P_{2 n-1} & =P_{2 n-1} L_{\zeta}, \\
P_{2}\left(P_{4} \ldots P_{2 n}\right) & =\left(P_{4} \ldots P_{2 n}\right) P_{2}, \\
P_{2} P_{4}\left(P_{6} \ldots P_{2 n}\right) & =\left(P_{6} \ldots P_{2 n}\right) P_{2} P_{4}, \\
& \vdots \\
P_{2} \ldots P_{2 n-2}\left(P_{2 n}\right) & =\left(P_{2 n}\right) P_{2} \ldots P_{2 n-2}, \\
L_{\eta}\left(P_{4} \ldots P_{2 n}\right) & =\left(P_{4} \ldots P_{2 n}\right) L_{\eta}, \\
L_{\eta}\left(P_{6} \ldots P_{2 n}\right) & =\left(P_{6} \ldots P_{2 n}\right) L_{\eta}, \\
& \vdots \\
L_{\eta} P_{2 n} & =P_{2 n} L_{\eta}
\end{aligned}\right\}
$$

Then, if the pairs $\left(L_{\zeta}, P_{1} P_{3} \ldots P_{2 n-1}\right)$ and $\left(L_{\eta}, P_{2} P_{4} \ldots P_{2 n}\right)$ are each owc, it follows that all $\left\{P_{i}\right\}$ and $\left\{L_{\zeta}\right\}$ have a unique common fixed point in $X$.

Corollary 4.2. Let $P_{1}, P_{2}, \ldots, P_{2 n}, A$ and $B$ be self mappings of a Menger space $(X, \mathcal{F}, \triangle)$ with $\triangle(a, a)=a$ for all $a \in[0,1]$ satisfying the following conditions:

(1) there exists $k \in(0,1)$ and $T \in \mathcal{T}$ such that

$$
F_{A x, B y}(k t) \geq\left(\min \left\{\begin{array}{c}
F_{P_{1} P_{3} \ldots P_{2 n-1} x, P_{2} P_{4} \ldots P_{2 n} y}(t), F_{A x, P_{1} P_{3} \ldots P_{2 n-1} x}(t), \\
F_{B y, P_{2} P_{4} \ldots P_{2 n} y}(t), \\
\triangle\left(F_{B y, P_{1} P_{3} \ldots P_{2 n-1} x}(\alpha t), F_{A x, P_{2} P_{4} \ldots P_{2 n} y}(2 t-\alpha t)\right)
\end{array}\right\}\right)^{h},
$$

for all $x, y \in X, \alpha \in(0,2), 0<h<1$ and $t>0$. 
(2) Suppose that

$$
\left\{\begin{aligned}
P_{1}\left(P_{3} \ldots P_{2 n-1}\right) & =\left(P_{3} \ldots P_{2 n-1}\right) P_{1}, \\
P_{1} P_{3}\left(P_{5} \ldots P_{2 n-1}\right) & =\left(P_{5} \ldots P_{2 n-1}\right) P_{1} P_{3}, \\
& \vdots \\
P_{1} \ldots P_{2 n-3}\left(P_{2 n-1}\right) & =\left(P_{2 n-1}\right) P_{1} \ldots P_{2 n-3}, \\
A\left(P_{3} \ldots P_{2 n-1}\right) & =\left(P_{3} \ldots P_{2 n-1}\right) A, \\
A\left(P_{5} \ldots P_{2 n-1}\right) & =\left(P_{5} \ldots P_{2 n-1}\right) A, \\
& \vdots \\
A P_{2 n-1} & =P_{2 n-1} A, \\
P_{2}\left(P_{4} \ldots P_{2 n}\right) & =\left(P_{4} \ldots P_{2 n}\right) P_{2}, \\
P_{2} P_{4}\left(P_{6} \ldots P_{2 n}\right) & =\left(P_{6} \ldots P_{2 n}\right) P_{2} P_{4}, \\
& \vdots \\
P_{2} \ldots P_{2 n-2}\left(P_{2 n}\right) & =\left(P_{2 n}\right) P_{2} \ldots P_{2 n-2}, \\
B\left(P_{4} \ldots P_{2 n}\right) & =\left(P_{4} \ldots P_{2 n}\right) B, \\
B\left(P_{6} \ldots P_{2 n}\right) & =\left(P_{6} \ldots P_{2 n}\right) B, \\
& \vdots \\
B P_{2 n} & =P_{2 n} B
\end{aligned}\right\}
$$

Then, if the pairs $\left(A, P_{1} P_{3} \ldots P_{2 n-1}\right)$ and $\left(B, P_{2} P_{4} \ldots P_{2 n}\right)$ are each owc, it follows that $P_{1}, P_{2}, \ldots, P_{2 n}, A$ and $B$ have a unique common fixed point in $X$.

Proof. The Corollary follows easily from Theorem 4.1, if we define $T\left(t_{1}, t_{2}, t_{3}, t_{4}, t_{5}\right)=t_{1}-\left(\min \left\{t_{2}, t_{3}, t_{4}, t_{5}\right\}\right)^{h}$ in Theorem 4.1 , for some $0<h<1$.

Corollary 4.3. Let $P_{1}, P_{2}, \ldots, P_{2 n}, A$ and $B$ be self mappings of a Menger space $(X, \mathcal{F}, \triangle)$ with $\triangle(a, a)=a$ for all $a \in[0,1]$ satisfying the following conditions:

(1) there exists $k \in(0,1)$ such that

$$
F_{A x, B y}(k t) \geq\left(F_{P_{1} P_{3} \ldots P_{2 n-1} x, P_{2} P_{4} \ldots P_{2 n} y}(t)\right)^{h},
$$

for all $x, y \in X, 0<h<1$ and $t>0$. 
(2) Suppose that

$$
\left\{\begin{aligned}
P_{1}\left(P_{3} \ldots P_{2 n-1}\right) & =\left(P_{3} \ldots P_{2 n-1}\right) P_{1}, \\
P_{1} P_{3}\left(P_{5} \ldots P_{2 n-1}\right) & =\left(P_{5} \ldots P_{2 n-1}\right) P_{1} P_{3}, \\
& \vdots \\
P_{1} \ldots P_{2 n-3}\left(P_{2 n-1}\right) & =\left(P_{2 n-1}\right) P_{1} \ldots P_{2 n-3}, \\
A\left(P_{3} \ldots P_{2 n-1}\right) & =\left(P_{3} \ldots P_{2 n-1}\right) A, \\
A\left(P_{5} \ldots P_{2 n-1}\right) & =\left(P_{5} \ldots P_{2 n-1}\right) A, \\
& \vdots \\
A P_{2 n-1} & =P_{2 n-1} A, \\
P_{2}\left(P_{4} \ldots P_{2 n}\right) & =\left(P_{4} \ldots P_{2 n}\right) P_{2}, \\
P_{2} P_{4}\left(P_{6} \ldots P_{2 n}\right) & =\left(P_{6} \ldots P_{2 n}\right) P_{2} P_{4}, \\
& \vdots \\
P_{2} \ldots P_{2 n-2}\left(P_{2 n}\right) & =\left(P_{2 n}\right) P_{2} \ldots P_{2 n-2}, \\
B\left(P_{4} \ldots P_{2 n}\right) & =\left(P_{4} \ldots P_{2 n}\right) B, \\
B\left(P_{6} \ldots P_{2 n}\right) & =\left(P_{6} \ldots P_{2 n}\right) B, \\
& \vdots \\
B P_{2 n} & =P_{2 n} B
\end{aligned}\right\}
$$

Then, if the pairs $\left(A, P_{1} P_{3} \ldots P_{2 n-1}\right)$ and $\left(B, P_{2} P_{4} \ldots P_{2 n}\right)$ are each owc, it follows that $P_{1}, P_{2}, \ldots, P_{2 n}, A$ and $B$ have a unique common fixed point in $X$.

Proof. The Corollary follows easily if we define $T\left(t_{1}, t_{2}, t_{3}, t_{4}, t_{5}\right)=$ $t_{1}-\left(t_{2}\right)^{h}$ in Theorem 4.1, for some $0<h<1$.

Corollary 4.4. Let $A, B, P$ and $Q$ be self maps of a Menger space $(X, \mathcal{F}, \triangle)$ with $\triangle(a, a)=a$ for all $a \in[0,1]$ satisfying the following condition:

(1) there exists $k \in(0,1)$ and $T \in \mathcal{T}$ such that

$$
T\left(\begin{array}{c}
F_{A x, B y}(k t), F_{P x, Q y}(t), F_{A x, P x}(t), F_{B y, Q y}(t), \\
\triangle\left(F_{B y, P x}(\alpha t), F_{A x, Q y}(2 t-\alpha t)\right)
\end{array}\right) \geq 0,
$$

for all $x, y \in X, \alpha \in(0,2)$ and $t>0$.

Then, if the pairs $(A, P)$ and $(B, Q)$ are each owc, it follows that $A, B, P$ and $Q$ have a unique common fixed point in $X$.

Proof. If we set $P_{1} P_{3} \ldots P_{2 n-1}=P$ and $P_{2} P_{4} \ldots P_{2 n}=Q$ in Theorem 4.1, then the result follows. 
Corollary 4.5. Let $A, B, P$ and $Q$ be self mappings of a Menger space $(X, \mathcal{F}, \triangle)$ with $\triangle(a, a)=a$ for all $a \in[0,1]$ satisfying the following condition:

(1) there exists $k \in(0,1)$ and $T \in \mathcal{T}$ such that

$$
\begin{aligned}
& F_{A x, B y}(k t) \geq\left(\min \left\{\begin{array}{c}
F_{P x, Q y}(t), F_{A x, P x}(t), F_{B y, Q y}(t), \\
\triangle\left(F_{B y, P x}(\alpha t), F_{A x, Q y}(2 t-\alpha t)\right)
\end{array}\right\}\right)^{h}, \\
& \text { for all } x, y \in X, \alpha \in(0,2), 0<h<1 \text { and } t>0 .
\end{aligned}
$$

Then, if the pairs $(A, P)$ and $(B, Q)$ are each owc, it follows that $A, B, P$ and $Q$ have a unique common fixed point in $X$.

Proof. The Corollary follows easily from Corollary 4.4, if we define $T\left(t_{1}, t_{2}, t_{3}, t_{4}, t_{5}\right)=t_{1}-\left(\min \left\{t_{2}, t_{3}, t_{4}, t_{5}\right\}\right)^{h}$ in Corollary 4.4 , for some $0<h<1$.

Now, we give an example which illustrates Corollary 4.4.

Example 4.1. Let $X=[0,2]$ with the metric $d$ defined by $d(x, y)=$ $|x-y|$ and for each $t \in[0,1]$, define

$$
F_{x, y}(t)= \begin{cases}\frac{t}{t+|x-y|}, & \text { if } t>0 ; \\ 0, & \text { if } t=0,\end{cases}
$$

for all $x, y \in X$. Clearly $(X, \mathcal{F}, \triangle)$ be a Menger space, where $\triangle(a, b)=$ $\min \{a, b\}$. Define the self mappings $A, B, P$ and $Q$ defined by

$$
\begin{aligned}
& A(x)=\left\{\begin{array}{ll}
x, & \text { if } 0 \leq x \leq 1 ; \\
2, & \text { if } 1<x \leq 2 .
\end{array} \quad P(x)= \begin{cases}1, & \text { if } 0 \leq x \leq 1 ; \\
0, & \text { if } 1<x \leq 2 .\end{cases} \right. \\
& B(x)=\left\{\begin{array}{ll}
1, & \text { if } 0 \leq x \leq 1 ; \\
2, & \text { if } 1<x \leq 2 .
\end{array} \quad Q(x)= \begin{cases}1, & \text { if } 0 \leq x \leq 1 ; \\
\frac{x}{2}, & \text { if } 1<x \leq 2 .\end{cases} \right.
\end{aligned}
$$

If we consider $T\left(t_{1}, t_{2}, t_{3}, t_{4}, t_{5}\right)=t_{1}-\left(\min \left\{t_{2}, t_{3}, t_{4}, t_{5}\right\}\right)^{h}$, for some $h, k \in(0,1)$, then the inequality

$$
F_{A x, B y}(k t) \geq\left(\min \left\{\begin{array}{c}
F_{P x, Q y}(t), F_{A x, P x}(t), F_{B y, Q y}(t), \\
\triangle\left(F_{B y, P x}(\alpha t), F_{A x, Q y}(2 t-\alpha t)\right)
\end{array}\right\}\right)^{h},
$$

is satisfied for all $x, y \in X$, for every $t>0$ and for every $\alpha \in(0,2)$. Clearly all the conditions of Corollary 4.4 are satisfied with respect to the distribution function $F_{x, y}$.

That is,

$$
\begin{aligned}
& A(1)=1=P(1) \text { and } A P(1)=1=P A(1), \\
& \text { and } \\
& B(1)=1=Q(1) \text { and } B Q(1)=1=Q B(1) .
\end{aligned}
$$


So, $A$ and $P$ as well as $B$ and $Q$ are owc mappings. Also 1 is the unique common fixed point of $A, B, P$ and $Q$. On the other hand, it is clear to see that the mappings $A, B, P$ and $Q$ are discontinuous at 1.

\section{Acknowledgement}

The authors are grateful to Professor Bessem Samet for the reprint of his valuable paper [23].

\section{References}

[1] C. T. Aage and J. N. Salunke, On fixed point theorems in fuzzy metric spaces, Int. J. Open Problems Compt. Math., 3(2) (2010), 123-131.

[2] M. Abbas and B. E. Rhoades, Common fixed point theorems for hybrid pairs of occaionally weakly compatible mappings satisfying generalized contractive condition of integral type, Fixed point Theory and Applications, (2007), Article ID 54101, 9 pages.

[3] M. Abbas and B. E. Rhoades, Common fixed point theorems for hybrid pairs of occasionally weakly compatible mappings defined on symmetric spaces, PanAmerican Math. J., 18(1) (2008), 55-62.

[4] M. Abbas and B. E. Rhoades, Common fixed point theorems for occasionally weakly compatible mappings satisfying a generalized contractive condition, Math. Comm., 13 (2008), 295-301.

[5] A. Aliouche and V. Popa, Common fixed point theorems for occasionally weakly compatible mappings via implicit relations, Filomat, 22(2) (2008), 99-107.

[6] A. Aliouche and V. Popa, General common fixed point theorems for occasionally weakly compatible hybrid mappings and applications, Novi Sad J. Math., 39(1) (2009), 89-109.

[7] M. A. Al-Thagafi, N. Shahzad, Generalized I-nonexpansive selfmaps and invariant approximations, Acta Math. Sinica, 24(5) (2008), 867-876.

[8] M. A. Al-Thagafi, N. Shahzad, A note on occasionally weakly compatible maps, Int. J. Math. Anal., 3(2) (2009), 55-58.

[9] I. Altun, H. A. Hancer and D. Turkoglu, A fixed point theorem for multi-maps satisfying an implicit relation on metrically convex metric spaces, Math. Commun., 11 (2006), 17-23.

[10] A. Bhatt, H. Chandra and D. R. Sahu, Common fixed point theorems for occasionally weakly compatible mappings under relaxed conditions, Nonlinear Analysis, 73 (2010), 176-182.

[11] H. Bouhadjera, A general common fixed point theorem for occasionally weakly compatible maps., An. Univ. Oradea, Fasc. Mat., 17(2) (2010), 17-22. 
[12] H. Bouhadjera and A. Djoudi, Common fixed point theorems of Greguš type for occasionally weakly compatible maps satisfying contractive conditions of integral type., An. Univ. Oradea, Fasc. Mat., 16 (2009), 145-152.

[13] H. Bouhadjera, A. Djoudi and B. Fisher, A unique common fixed point theorem for occasionally weakly compatible maps, Surveys in Mathematics and its Applications, 3 (2008), 177-182.

[14] H. Bouhadjera, A. Djoudi and B. Fisher, A unique common fixed point theorem for occasionally weakly compatible maps, Bull. Allahabad Math. Soc., 24(1) (2009), 1-6.

[15] H. Bouhadjera, C. Godet-Thobie, Common fixed point theorems for occasionally weakly compatible single and set-valued maps, HAL-00273238, Version 1, 15 April 2008 .

[16] H. Bouhadjera, C. Godet-Thobie, Common fixed point theorems for occasionally weakly compatible maps, arXiv: 0812.3734v2 [math. FA], 17 June 2009.

[17] H. Chandra, A. Bhatt, Fixed point theorems for occasionally weakly compatible maps in probabilistic semi-metric space, Int. J. Math. Anal., 3(12) (2009), 563570 .

[18] S. S. Chang, Y. J. Cho, S. M. Kang, Probabilistic Metric Spaces and Nonlinear Operator Theory, Sichuan Univ. Press (Chengdu), 1994.

[19] S. S. Chang, Y. J. Cho, S. M. Kang, Nonlinear Operator Theory in Probabilistic Metric Spaces, Nova Science Publishers, Inc., New York, 2001.

[20] S. Chauhan and S. Kumar, Fixed point theorems in Menger spaces and applications to metric spaces, J. Appl. Math. Inform., 30(5-6) (2012), 729-740.

[21] S. Chauhan, S. Kumar and B. D. Pant, Common fixed point theorems for occasionally weakly compatible mappings in Menger spaces, J. Adv. Research Pure Math., 3(4) (2011), 17-23.

[22] S. Chauhan and B. D. Pant, Common fixed point theorems for occasionally weakly compatible mappings using implicit relation, J. Indian Math. Soc. (N.S.), 77(1-4) (2010), 13-21.

[23] Lj. B. Ćirić, B. Samet and C. Vetro, Common fixed point theorems for families of occasionally weakly compatible mappings, Math. Comp. Model., 53 (2011), 631-636.

[24] O. Hadžić and E. Pap, Fixed point theory in probabilistic metric spaces, Dordrecht: Kluwer Academic publishers, 2001.

[25] M. Imdad, S. Kumar and M. S. Khan, Remarks on some fixed point theorems satisfying implicit relations, Rad. Math., 11 (2002), 135-143.

[26] G. Jungck, B. E. Rhoades, Fixed points for set valued functions without continuity, Indian J. Pure Appl. Math., 29(3) (1998), 227-238. 
[27] G. Jungck, B. E. Rhoades, Fixed point theorems for occasionally weakly compatible mappings, Fixed Point Theory, 7 (2006), 286-296.

[28] G. Jungck, B. E. Rhoades, Fixed point theorems for occasionally weakly compatible mappings, Fixed Point Theory, 9 (2008), 383-384. (Erratum)

[29] M. A. Khan and Sumitra, Common fixed point theorems for occasionally weakly compatible maps in fuzzy metric spaces, Far East J. Math. Sci., 41(2) (2009), 285-293.

[30] K. Menger, Statistical metrics, Proc. Nat. Acad. Sci. U.S.A., 28 (1942), 535-537.

[31] S. N. Mishra, Common fixed points of compatible mappings in PM-spaces, Math. Japon., 36 (1991), 283-289.

[32] B. D. Pant, M. Abbas and S. Chauhan, Coincidences and common fixed points of weakly compatible mappings in Menger spaces, J. Indian Math. Soc. (N.S.), 80(1-4) (2013), 127-139.

[33] B. D. Pant and S. Chauhan, Common fixed point theorem for occasionally weakly compatible mappings in Menger space, Surv. Math. Appl., 6 (2011), 1-7.

[34] B. D. Pant and S. Chauhan, Fixed points of occasionally weakly compatible mappings using implicit relation, Commun. Korean Math. Soc., 27(3) (2012), 513522 .

[35] B. D. Pant and S. Chauhan, Fixed point theorems for occasionally weakly compatible mappings in Menger spaces, Mat. Vesnik, 64(4) (2012), 267-274.]

[36] B. D. Pant, S. Chauhan and B. Fisher, Fixed point theorems for families of occasionally weakly compatible mappings, J. Indian Math. Soc. (N.S.), 79(1-4) (2012), 127-138.

[37] V. Popa, Some fixed point theorems for compatible mappings satisfying an implicit relation, Demonsratio Math., 32 (1999), 157-163.

[38] V. Popa, A general coincidence theorem for compatible multivalued mappings satisfying an implicit relation, Demonsratio Math., 33 (2000), 159-164.

[39] K. P. R. Sastry, G. A. Naidu, P. V. S. Prasad, V. M. Latha, S. S. A. Sastry, A critical look at fixed point theorems for occasionally weakly compatible maps in probabilistic semi-metric spaces, Int. J. Math. Anal., 4(27) (2010), 1341-1348.

[40] B. Schweizer, A. Sklar, Statistical metric spaces, Pacific J. Math., 10 (1960), 313-334.

[41] B. Schweizer, A. Sklar, Probabilistic Metric Spaces, Elsevier, North Holland, New York, 1983.

[42] V. M. Sehgal and A. T. Bharucha-Reid, Fixed points of contraction mappings on probabilistic metric spaces, Math. Systems Theory, 6 (1972), 97-102.

[43] S. Sedghi, K. P. R. Rao and N. Shobe, A general common fixed point theorem for multi-maps satisfying an implicit relation on fuzzy metric spaces, Filomat, 22(1) (2008), 1-11. 
[44] S. Sharma and B. Deshpande, On compatible mappings satisfying an implicit relation in common fixed point consideration, Tamkang J. Math., 33 (2002), 245-252.

[45] B. Singh, S. Jain, A fixed point theorem in Menger space through weak compatibility, J. Math. Anal. Appl., 301 (2005), 439-448.

[46] C. Vetro, Some fixed point theorems for occasionally weakly compatible mappings in probabilistic semi-metric spaces, Int. J. Modern Math., 4(3) (2009), 277-284.

Sunny Chauhan

R. H. Government Postgraduate College,

Kashipur-244 713, (U.S. Nagar), Uttarakhand, India.

E-mail: sun.gkv@gmail.com

M. Alamgir Khan

Department of Natural Resources Engineering and Management, University of Kurdistan,

Hewler, Iraq.

E-mail: alam3333@gmail.com

B. D. Pant

Government Degree College,

Champawat-262 523, Uttarakhand, India.

E-mail: badridatt.pant@gmail.com 